\title{
VLBA Observations of 96 Sources in the Extended Chandra Deep Field South
}

\author{
E. Middelberg ${ }^{* a}$, H. Rottmann ${ }^{b}$, W. Alef ${ }^{b}$, S. Tingay ${ }^{c}$, A. Deller ${ }^{d}$, R. Norris ${ }^{e}$, and \\ E. Lenc ${ }^{e}$
}

${ }^{a}$ Astronomisches Institut der Universität Bochum

middelbergeastro.rub. de

${ }^{b}$ Max-Planck-Institut für Radioastronomie

rottmann, walef@mpifr-bonn.mpg.de

${ }^{c}$ Department of Imaging and Applied Physics, Curtin University of Technology

s.tingayaivec.org

${ }^{d}$ Centre for Astrophysics and Supercomputing, Swinburne University of Technology

adellerdastro.swin.edu. au

${ }^{e}$ Australia Telescope National Facility

ray.norris, emil.lencecsiro.au

\begin{abstract}
Sensitive radio observations of extragalactic fields yield important boundary conditions to study the evolution of galaxies throughout cosmic time. One of the main goals is to separate the energy output from accretion in Active Galactic Nuclei from that in actively star-forming galaxies. VLBI observations can filter out low brightness temperature objects from such surveys since they are sensitive only to the very high brightness temperatures in AGN. However, it is technically challenging to image objects spread out across fields more than a few arcminutes in extent. Here we describe a project to observe simultaneously 96 radio sources located within the primary beam of the antennas, to search for AGN activity and image their radio jets.
\end{abstract}

The 9th European VLBI Network Symposium on The role of VLBI in the Golden Age for Radio Astronomy and EVN Users Meeting

September 23-26, 2008

Bologna, Italy

\footnotetext{
* Speaker.
} 


\section{Introduction}

We have conducted the Australia Telescope Large Area Survey (ATLAS) of the Chandra Deep Field South (CDFS) and European Large Area ISO Survey - South 1 (ELAIS) regions at $1.4 \mathrm{GHz}$, with a resolution of around $7 \mathrm{arcsec}$. The aim is to produce the widest ( $7 \mathrm{deg}^{2}$ total area) deep ( $15 \mu \mathrm{Jy} \mathrm{rms}$ ) radio survey ever attempted. The survey areas were chosen to cover the Southern SWIRE (Spitzer Wide-area InfraRed Extragalactic survey) fields, which have deep optical, nearinfrared, and far-infrared (and in some parts of the field, deep X-ray) data. The broad scientific goals are to understand the evolution of actively star-forming and passively evolving galaxies in the early Universe; the spatial distribution and clustering of evolved galaxies, starbursts, and AGN at $0.5<z<3$; and the relative contributions to the cosmic background from accretion energy and nucleosynthesis energy. The first catalogues have been published $([1,2])$, and relevant results are beginning to emerge $([3,4,5])$.

The motivations for a VLBI survey of all sources in this field are threefold: (i) To find AGN activity. Identifying the processes which contribute to a galaxy's energy budget requires us to determine whether or not it contains an AGN. VLBI observations are sensitive only to very high brightness temperatures of the order of $10^{6} \mathrm{~K}$ and higher, which is an almost unambiguous sign for AGN activity. Information from other sources (e.g. spectral index, arcsec-scale morphology, radio luminosity, polarization, etc.) can then help to strengthen the argument for or against the existence of an AGN. (ii) To image AGN jets as a function of redshift. A subclass of the sources we found in the Australia Telescope Compact Array (ATCA) observations have signatures of starbursts in their spectral energy distributions, but AGN-like morphologies even on arcsec scales (e.g., triple radio galaxies). But most objects are unresolved, and so we have no information about their structures. How many more of these objects have we missed because we do not know their sub-arcsec morphologies? Are they common? Is there a significant population of mixed AGN/starbursts at high redshifts? VLBI observations of our sample of galaxies will result in an unprecedented database of AGN and jet morphologies, which can be investigated as a function of redshift and infrared host galaxy properties. (iii) To Develop wide-field VLBI techniques. This project is a first-class technical challenge. While there have been a few projects to image large fields $([6,7])$, imaging of hundreds of sources across several square degrees is only just becoming possible and has so far been restricted to sub-GHz frequencies. The computational load required for this type of imaging task is vast, and separation between correlation and imaging will become blurred.

To prepare the full survey and explore the necessary correlation and calibration strategies we have carried out a pilot project which we describe in this article.

\section{Observations and correlation}

\subsection{Observations}

On 3 July 2007 we have observed with the Very Long Baseline Array (VLBA) at $1.4 \mathrm{GHz}$ a single pointing centred on the original CDFS (Figure 1). We used 8 dual-polarization IFs with $8 \mathrm{MHz}$ bandwidth each and two-bit sampling, resulting in a recording bitrate of $512 \mathrm{Mbps}$, which was the highest possible bitrate of the VLBA at that time. The low declination of $-27^{\circ}$ allowed us to observe the target for $9 \mathrm{~h}$ only, yielding a total of 167 baseline-hours from which we expect 

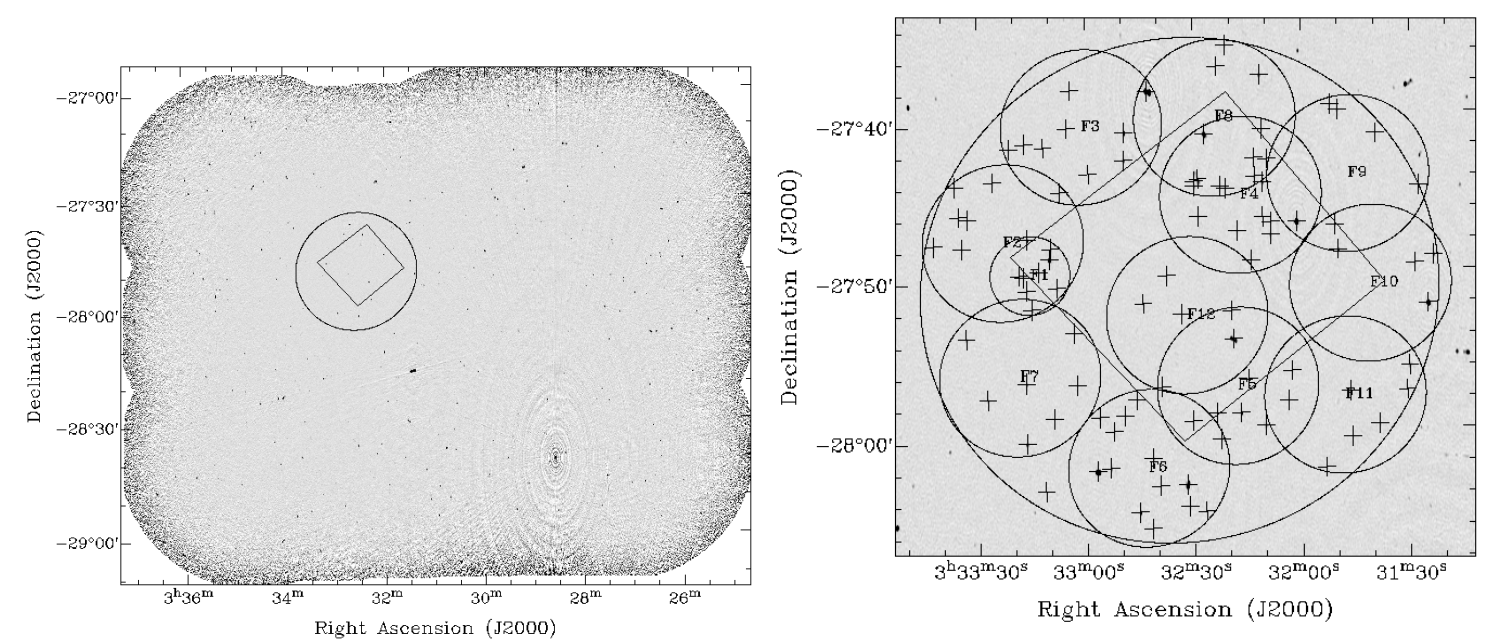

Figure 1: Left panel: The region imaged with the ATCA at $1.4 \mathrm{GHz}$. The circle indicates the FWHM of the antennas and the rectangle denotes the locations of the original CDFS. Right panel: A magnified view of the circled region of the left panel. Crosses indicate radio sources found in the ATCA observations, and circles indicate regions across which the amplitude losses due to time and bandwidth smearing are kept below $10 \%$ in correlation (see text). The small circle at around 00:33:15, -27:48:00 denotes a test area with lower resolution which has been used to explore the correlation strategy.

image sensitivities of $35 \mu \mathrm{Jy}$. A six-minute phase reference cycle time was used, with 5 min allocated to the target and $1 \mathrm{~min}$ to the nearby strong $(600 \mathrm{mJy})$ calibrator source J0348-2749. Short observations of the fringe finders 3C454.3 and 4C39.25 were inserted every two hours. The data were recorded on disk and shipped to the Max-Planck-Institut für Radioastronomie for correlation.

\subsection{Correlation}

Correlation was carried out on a 22-node computer cluster with 176 compute cores using the DiFX software correlator ([8]). Our general approach to make images of the known locations of the 96 sources was as follows: (i) correlate with a resolution high enough to suppress time and bandwidth smearing in a given area, (ii) calculate delays and phases for each of the sources in that area, calculate the difference to the phase centre and apply the difference to the data, (iii) average in time and frequency and make an image.

Time averaging and bandwidth smearing (e.g., [9]) cause the amplitudes of sources away from the phase centre to drop. This can be avoided by decreasing the time averaging interval, $\tau$, and the channel bandwidth, $\Delta v$, in correlation, at the expense of a larger data set. Somewhat arbitrarily, we decided that we wanted to keep amplitude losses on a $5000 \mathrm{~km}$ baseline below $10 \%$. We note that only $5 \%$ of the baselines in our observations are longer than that, so the effects of smearing on an image should then be very small.

To limit the effects of bandwidth-smearing (a form of chromatic aberration) and time-average smearing to below $10 \%$ at the edge of the primary beam ( 15 arcmin from the phase centre) would require integration times of $\sim 50 \mathrm{~ms}$ and a spectral resolution better than $4 \mathrm{kHz}$. This would result in $3 \mathrm{~TB}$ of visibility data which would have been very difficult to manage. We therefore decided to correlate multiple times with lower resolution, resulting in smaller data sets. The 12 large circles in 
Figure 1 represent fields with 5 arcmin radius in which the losses due to smearing effects stay below $10 \%$, using $\tau=0.25 \mathrm{~s}$ and $\Delta v=15.6 \mathrm{kHz}$. Each of these 12 correlator passes resulted in a $216 \mathrm{~GB}$ data set, which still is large, but manageable. We refer to these data sets as the high-resolution, or HR, data sets in this article.

We also correlated with lower resolution $(\tau=2 \mathrm{~s}, \Delta v=123 \mathrm{kHz})$ using the coordinates of the strong source S503. This low-resolution (LR) data set was used for calibration, since its considerable smaller size allowed self-calibration and other tasks to be carried out in much shorter times. Phase and amplitude calibration yield antenna-based corrections to the raw visibility amplitudes and so are independent of the temporal or spectral resolution of the data. Only the bandpass calibration and some flagging due to filter roll-off at the band edges needed to be repeated on the HR data sets, to account for the larger number of channels.

\subsection{Calibration and imaging}

We derived the complex gains with the following steps, using the LR data set containing S503 and the Astronomical Image Processing System (AIPS): Amplitude calibration was carried out with measurements of system temperature and known antenna gains. Ionospheric dispersive delays were corrected using a global model of ionospheric total electron content. Instrumental delays were derived using fringe-fitting of calibrator data, keeping the IFs and polarizations separate, and using a $10 \sigma$ cutoff. These corrections were extrapolated to the entire data set. Then fringe-fitting using band- and polarization-averaged data was carried out on J0348-2749 to derive phase corrections. The J0348-2749 data were exported to Difmap ([10]) for detailed imaging and (amplitude) selfcalibration. We used a modified version of Difmap to export the Difmap phase and amplitude corrections to AIPS, where they were applied to S503. S503 was then imaged in AIPS and one cycle of phase self-calibration was used to obtain a good image. This yielded a set of delay, amplitude, and phase corrections which we copied to the HR data sets for imaging. Bandpass calibration was carried out using the HR data set to account for the higher number of channels, and flagging of $10 \%$ of the bandpass was also done using the HR data set, for the same reason. The calibration was then applied to the visibilities of the HR data set.

VLBA observations in general do not need to be corrected for primary beam attenuation, since in the vast majority of cases the targets are on-axis and the attenuation is negligible. In our observations, primary beam attenuation can be as high as $50 \%$ and needs correction. However, this is not trivial since the VLBA suffers from noticeable $\left(>1.5^{\prime}\right)$ beam squint, i.e., the RCP and LCP beams have a significant separation on the sky. Hence a source away from the optical axis will wander through the two beams on a trajectory which is not concentric with respect to the RCP and LCP beam axes, resulting in a somewhat complicated, baseline and time dependent pattern of attenuation throughout the observations. As a result, the amplitudes measured in RCP and LCP can differ by up to $5 \%$; the images presented here have not been corrected for this effect.

For each source within the correlated field of view the $(u, v, w)$ coordinates of the calibrated data set were recalculated using the AIPS task UVFIX. The data were then averaged in time into $2 \mathrm{~s}$ intervals and in each IF. In Difmap, a $4096^{2}$ pixel image was made of these data sets using a cell size of 1 mas, and was searched for emission. If emission was detected, one cycle of phase self-calibration was used to finalize the images. 

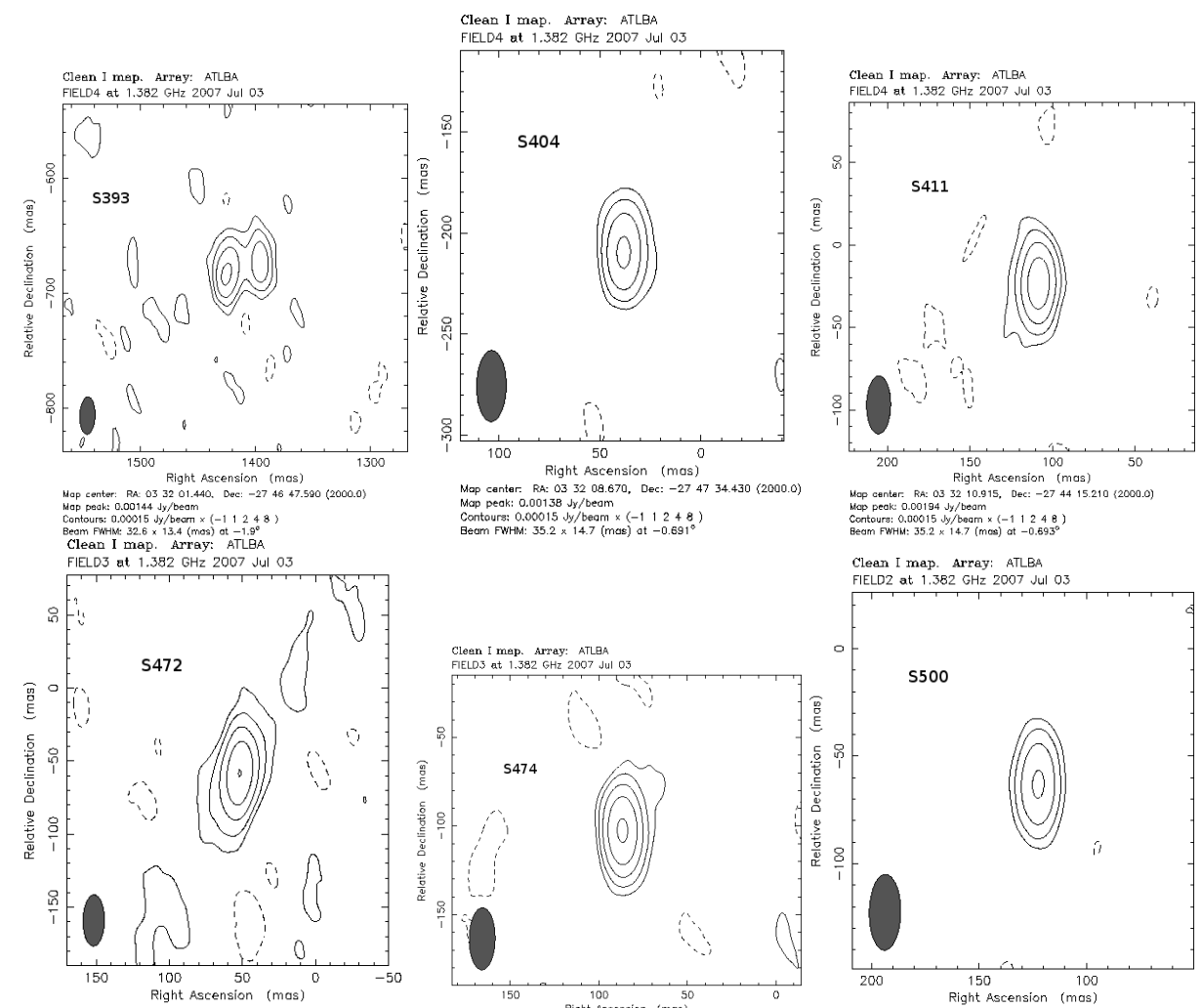

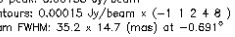

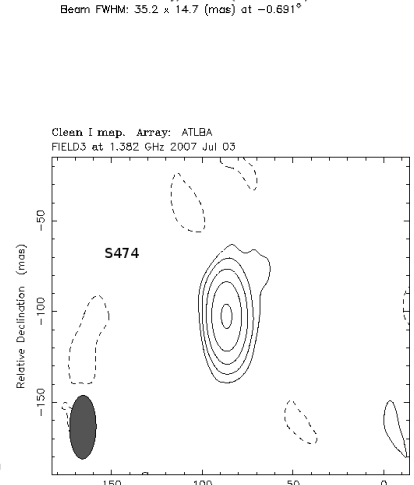

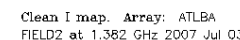

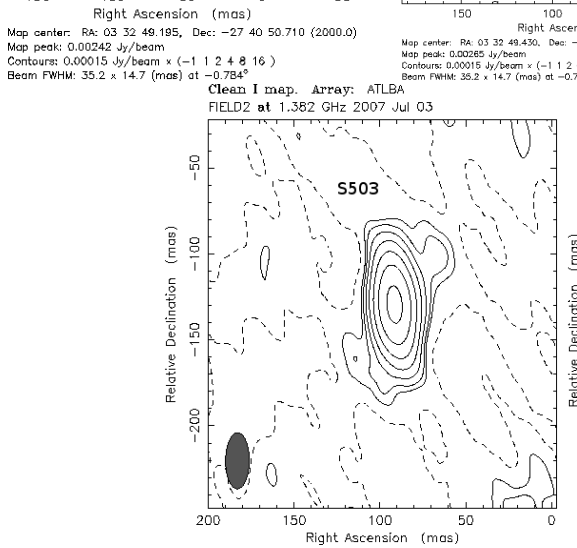

Right Ascension (mas)
Map cantar: Ras 033 10.191, Dec: $-274842.060(2000)$

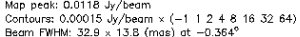
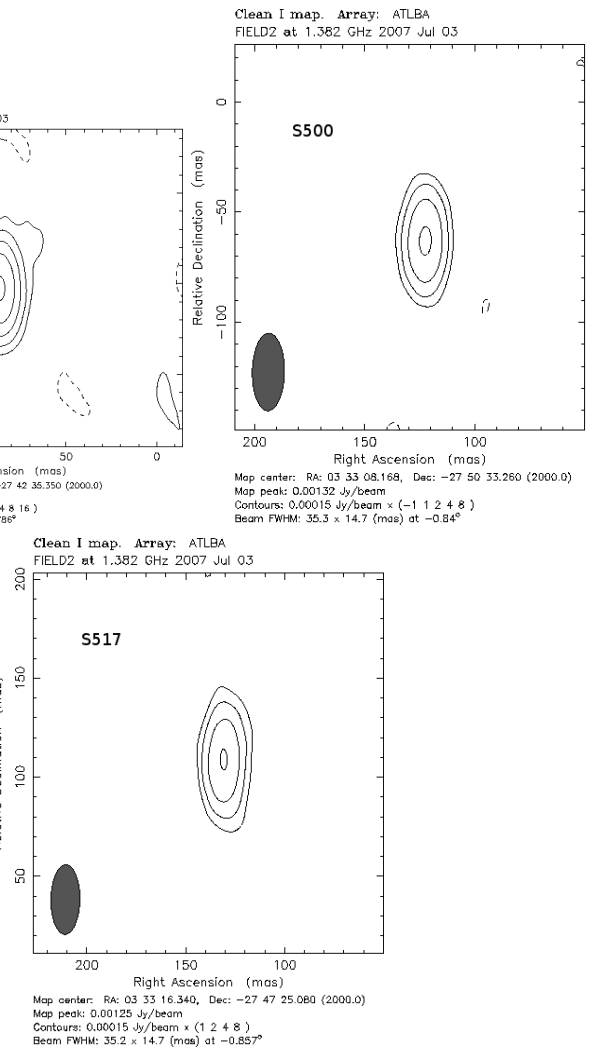

Figure 2: Contour plots of the 8 detected sources in the correlated Fields 2,3, and 4. Source names taken from [1] are given in the plots. Contours begin at $150 \mu \mathrm{Jy}_{\text {beam }}^{-1}$ and increase by factors of two.

\section{Results}

At the time of writing, three fields had been processed (Fields 2, 3, and 4). These fields together contain 44 sources, 8 of which were detected. Contour plots of these sources are shown in Figure 2. The images were made using natural weighting, and a Gaussian taper with a magnitude of 0.1 at a $(u, v)$ distance of $20 \mathrm{M} \lambda$ was applied. Some source properties are given in Table 1.

The results presented here are the first, tentative results from our observations, and a number of inconsistencies still need investigation. For example, we find that the source positions derived 


\begin{tabular}{lllcc}
\hline \hline Source & RA & Dec & $\begin{array}{c}S_{1.4, \text { ATCA }} \\
\mathrm{mJy}\end{array}$ & $\begin{array}{c}S_{1.4, \text { VLBA }} \\
\mathrm{mJy}\end{array}$ \\
\hline S393 & $03: 32: 01.440$ & $-27: 46: 47.59$ & 49.1 & 3.9 \\
S404 & $03: 32: 08.670$ & $-27: 47: 34.43$ & 1.7 & 1.4 \\
S411 & $03: 32: 10.915$ & $-27: 44: 15.21$ & 2.7 & 2.4 \\
S472 & $03: 32: 49.195$ & $-27: 40: 50.71$ & 4.7 & 4.1 \\
S474 & $03: 32: 49.430$ & $-27: 42: 35.35$ & 3.2 & 2.9 \\
S500 & $03: 33: 08.168$ & $-27: 50: 33.26$ & 1.0 & 1.3 \\
S503 & $03: 33: 10.191$ & $-27: 48: 42.06$ & 19.3 & 17.6 \\
S517 & $03: 33: 16.340$ & $-27: 47: 25.08$ & 2.4 & 1.6 \\
\hline
\end{tabular}

Table 1: Parameters of the detected sources. Coordinates are the observed coordinates based on the ATCA source positions. The VLBI images in Figure 2 are labelled relative to these coordinates. Flux density errors are $20 \%$ for the ATCA observations, and similar for the VLBA observations, but we have not yet accurately estimated these. Also note that the VLBA flux densities are not corrected for primary beam attenuation.

from data with re-computed $(u, v, w)$ coordinates differ from positions derived from data correlated in a normal fashion by as much as 15 mas. This also occurs when the re-calculation of $(u, v, w)$ is carried out outside AIPS, and the cause of this effect is yet to be found. An interesting preliminary result is that five of the eight detected sources also have X-ray detections.

\section{Conclusions}

We have carried out wide-field VLBI observations of the Chandra Deep Field South, to image 96 targets in an area covered by the primary beam of the VLBA antennas. We have developed a correlation and imaging strategy to enable handling of the huge data volume on standard computing equipment. Amplitude losses due to time averaging and bandwidth smearing have been reduced to below $10 \%$ by chosing an appropriate temporal and spectral resolution. The three data sets processed so far allowed us to image 44 sources, 8 of which have been detected.

\section{References}

[1] R. P. Norris, J. Afonso, P. N. Appleton, et al. AJ, 132:2409-2423, December 2006.

[2] E. Middelberg, R. P. Norris, T. J. Cornwell, et al. AJ, 135:1276-1290, April 2008.

[3] B. J. Boyle, T. J. Cornwell, E. Middelberg, et al. MNRAS, 376:1182-1188, April 2007.

[4] R. P. Norris, S. J. Tingay, C. Phillips, et al. MNRAS, 2007.

[5] E. Middelberg, R. P. Norris, S. Tingay, et al. A\&A, 491:435-439, November 2008.

[6] E. Lenc, M. A. Garrett, O. Wucknitz, et al. ApJ, 673:78-95, January 2008.

[7] M. A. Garrett, J. M. Wrobel, and R. Morganti. ApJ, 619:105-115, January 2005.

[8] A. T. Deller, S. J. Tingay, M. Bailes, et al. PASP, 119:318-336, March 2007.

[9] A. H. Bridle and F. R. Schwab. In G. B. Taylor, C. L. Carilli, and R. A. Perley, editors, Synthesis Imaging in Radio Astronomy II, volume 180 of ASP Conference Series, page 371, 1999.

[10] M. C. Shepherd. In ASP Conf. Ser. 125: Astronomical Data Analysis Software and Systems VI, page 77, 1997. 